\title{
Hiperintensidad en secuencias T2 y tamaño como factores pronósticos de obliteración en malformaciones arteriovenosas tratadas mediante radiocirugía
}

\author{
I. Arrese; R. Alday; P.A. González; J. Campollo*; A. Ramos*; P. Dominguez**; J.P. Fernández-Letón; A. Pérez- \\ Núñez y R. D. Lobato
}

Servicios de Neurocirugía, Neuroradiologia*, Oncología Radioterápica** y Radiofísica***. Hospital Universitario 12 de Octubre. Madrid.

\section{Resumen}

Objetivo. Analizar retrospectivamente los factores implicados en la obliteración de las malformaciones arteriovenosas (MAVs) tratadas mediante radiocirugía.

Métodos. Se revisaron retrospectivamente las historias clínicas y las pruebas de imagen de 59 pacientes con MAVs tratados consecutivamente mediante tratamiento radioquirúrgico en nuestro Servicio. Se recogen los datos demográficos y clínicos en el momento del diagnóstico, así como los referentes al tratamiento empleado y la evolución de dichos pacientes.

Resultados. Se consiguió la obliteración en el $\mathbf{7 7 \%}$ de las MAVs tratadas. La obliteración completa se observó en los primeros tres años en sólo el $40 \%$ de los casos, mientras que en el resto, la obliteración ocurrió entre los tres y los cinco años. Diez pacientes (17\%) sufrieron complicaciones agudas o crónicas. Sólo un paciente murió como resultado de una hemorragia intraparenquimatosa durante el periodo de seguimiento. El análisis multivariable utilizando los diversos factores y parámetros potencialmente relacionados con la obliteración mostró que sólo la hiperintensidad perilesional observada en secuencias $T 2$ de la RM y un tamaño del nidus menor de $3 \mathrm{~cm}$ incrementaron de manera estadísticamente significativa la probabilidad de oclusión completa $(p=0,03$ y $p=0,05$, respectivamente).

Conclusión. Nuestros resultados, son similares a los reportados en otras series. Sin embargo, se obtuvo una menor tasa de oclusiones en las MAVs $>3 \mathrm{~cm}$ de diámetro, confirmando que el tamaño es un factor determinante en probabilidad de cierre de las MAVs tratadas radioquirúrgicamente. El desarrollo de hiperintensidad perilesional en secuencias $T 2$ de las RMs durante el seguimiento, fue el factor que se relacionó de manera más directa con una mayor probabilidad de oclusión. Un alto número de obliteraciones ocurrieron más de 3 años después del tratamiento, por lo que en presencia de

Recibido: 22-04-08. Aceptado: 10-11-08 restos pequeños de nidus, podría mantenerse una actitud expectante durante un mayor periodo de tiempo.

PALABRAS CLAVE: Radiocirugía. Malformaciones arteriovenosas. RM. Factores pronósticos. Hiperintensidad

Hyperintensity on T2 MRI and size as predictors of obliteration in radiosurgically treated arteriovenous malformations

Sumary

Objetive. The aim of this study was to determine which factors were statistically related to radiological and clinical outcomes following radiosurgical treatment of arteriovenous malformations (AVMs).

Methods. The data of 59 patients receiving radiosurgical treatment at our department were retrospectivelly reviewed. Different clinical and biological data, including Spetzler-Martin grade, the presentation of symptoms, radiation dose, number of isocenters and both radiological and clinical outcome, were subjected to multivariate analysis.

Results. AVM obliteration was achieved in $77 \%$ of patients, the majority of them occurring between 3-5 years after treatment. Ten patients $(17 \%)$ showed either acute or delayed complications. Only one patient died due tor hemorrhage during the follow-up after radiosurgery. A multivariate analysis showed that, hyperintensity on T2 MRI and a nidus smaller than $3 \mathrm{~cm}$ were the only factors statistically related to oclusion of the $\operatorname{AVM}(\mathbf{p}=0,03$ and $\mathbf{p}=0,05$, respectively).

Conclusion. The nidus size and the development of hyperintensity on T2 MRI after the treatment were the strongest predictive factors of obliteration in our series of AVMs radiosurgically treated. Moreover, given that many AVMs showed complete obliteration between 3-5 years after treatment, we recommend to wait untill 5 years after treatment before considering a new terapeuthic approach in patients showing small residual nidus at control imaging. 
KEY WORDS: Radiosurgery. Arteriovenous malformation. MRI. Prognostic factors. Hyperintensity.

\section{Introducción}

La radiocirugía es una opción terapeútica ampliamente aceptada en el tratamiento de las malformaciones arteriovenosas (MAVs), por lo que han sido múltiples las series publicadas que reportan tasas de oclusión del 60$80 \%$ de los pacientes tratados ${ }^{8,11,13,19}$. Por ello, aunque la resección microquirúrgica continúa siendo el método más resolutivo a la hora de eliminar definitivamente el riesgo de sangrado, la radiocirugía puede ser un tratamiento alternativo en pacientes con MAVs de pequeño y mediano tamaño $\mathrm{y}$ alto riesgo quirúrgico.

Sin embargo, el tratamiento radioquirúrgico de las MAVs plantea dos problemas principales. Por una parte, el fracaso por falta de oclusión completa en un $20-40 \%$ de los casos y, sobre todo, la persistencia del riesgo de sangrado después de la aplicación del tratamiento hasta la consecución del cierre completo del nidus. Por este motivo, resulta de gran interés determinar si existen factores predictivos que nos permitan determinar la probabilidad de oclusión en las MAVs tratadas con radiocirugía. Con este propósito hemos revisado retrospectivamente nuestra experiencia con este tipo de tratamiento acumulada en los últimos 6 años.

\section{Tabla 1}

Datos biológicos y clínicos en una serie de 59 pacientes con MAVs tratadas con radiocirugía

\begin{tabular}{lc}
\hline Factor & Casos $\mathrm{N}^{\circ}(\%)$ \\
\hline $\begin{array}{l}\text { Edad (años) } \\
>60\end{array}$ & $5(8.5)$ \\
$<60$ & $54(91.5)$ \\
Sexo & \\
$\quad$ Mujer & $30(50.8)$ \\
Hombre & $29(49.2)$ \\
& \\
Presentación clínica & \\
$\quad$ Hemorragia intracraneal & $23(39)$ \\
Crisis convulsiva & $15(25.4)$ \\
Karnofsky & \\
$>70$ & $49(83)$ \\
$\quad<70$ & $10(17)$ \\
Tratamiento previo & \\
Embolización & $14(23.7)$ \\
Resección subtotal & $14(23.7)$ \\
\hline
\end{tabular}

\section{Pacientes y métodos}

Hemos revisado retrospectivamente 59 pacientes con MAVs tratados mediante radiocirugía en nuestro centro desde Febrero de 1997 hasta Marzo del 2004. Ninguno de ellos había recibido tratamiento radioquirúrgico previo. La Tabla 1 muestra las características demográficas de los 59 pacientes. Todos los casos fueron valorados antes de recibir el tratamiento por un equipo formado por neurocirujanos, radioterapeutas, radiofísicos y neurorradiólogos intervencionistas.

El tratamiento se realizó mediante la colocación de marco esterotáxico BRW (Brown-Robert-Wells) y software para planificación radioquirúrgica Radionics. El tratamiento se administró mediante LINAC (Saturno $6 \mathrm{Mv}$ ).

El seguimiento de base se realizó mediante angio-RM (ARMs) periódicas. En los casos en los que no se observaba restos de nidus en la ARM de control la obliteración fue confirmada mediante arteriografía.

En este estudio se definió la curación como la obliteración completa demostrada mediante angiografía. Las complicaciones fueron clasificadas como: hemorragia, déficit neurológico permanente o transitorio adicional al que presentara el paciente previo al tratamiento, aparición de crisis postratamiento, aumento de la frecuencia de las crisis, aparición de edema con efecto de masa, radionecrosis y desarrollo de lesiones quísticas. Las variables independientes potencialmente relacionadas con la evolución analizadas fueron la edad, el sexo, el diámetro del nidus, la elocuencia del área, la presencia o no de drenaje profundo, el grado de Spetzler-Martin, la dosis, el número de isocentros utilizados, los tratamientos previos embolizadores o quirúrgicos y el desarrollo de hiperintensidad en secuencias T2 en la RM. Todas las imágenes radiológicas (ARMs y arteriografías) fueron revisadas por un neurocirujano y un neurorradiólogo, a fin de reevaluar las variables radiológicas.

Para determinar los posibles factores relacionados con la posibilidad de oclusión se realizó un estudio univariable, curvas de supervivencia de Kaplan-Meier y un análisis multivariable mediante regresión de Cox. La significación estadística vino definida por un valor de $\mathrm{p}<0.05$. Los análisis estadísticos se realizaron con el paquete informático SPSS. 10.

\section{Resultados}

Los hallazgos en las pruebas de imagen de nuestra serie quedan reflejados en la Tabla 2. Los datos más destacables son la alta proporción de MAVs menores de 3 centímetros de diámetro $(69.5 \%)$ y de localización en zonas elocuentes (67.8\%), lo que se traduce en que un $64.4 \%$ de las MAVs fueron grados de Spetzler-Martin 2 y 3. Los datos dosimétricos quedan reflejados en la Tabla 3. 
Tabla 2

Hallazgos radiológicos en 59 pacientes con MAVs tratadas mediante radiocirugía

\begin{tabular}{lcccr}
\hline & \multicolumn{1}{c}{ Casos $\mathrm{n}^{\circ}(\%)$} & \multicolumn{2}{c}{ Spetzler-Martin } \\
\cline { 2 - 4 } & & & & \\
Diámetro $<3 \mathrm{~cm}$ & $41(69.5 \%)$ & I & $12(20.3 \%)$ \\
Área elocuente & $40(67.8 \%)$ & III & $20(33.9 \%)$ \\
Drenaje profundo & $26(44.1 \%)$ & IV & $6(10.5 \%)$ \\
& & V & $5(5.1 \%)$
\end{tabular}

Localización del nidus:

$\begin{array}{lc}\text { Lobar supratentorial } & 35(59.3 \%) \\ \text { Profundo supratentorial } & 22(37.3) \\ \text { Infratentorial } & 2(5.1)\end{array}$

Hiperintensidad perilesional en T2-MR

$17(29 \%)$

Tabla 3

Datos dosimétricos del tratamiento radioquirúrgico en 59 pacientes tratados con MAVs

Diámetro del nidus (cms): $0.8 \mathrm{~cm}-4.3 \mathrm{~cm}$ (media $2.4 \mathrm{~cm}$ )

$\begin{array}{cc}\mathrm{N}^{\mathrm{o}} \text { de isocentros: } & {\operatorname{Casos~} \mathrm{n}^{\circ}(\%)} \\ \text { Uno } & 54(92.5 \%) \\ \text { Dos } & 4(5.5 \%) \\ \text { Tres } & 1(2 \%)\end{array}$

Dosis al margen

$\begin{array}{lr}15 \text { Gy } & 45(76 \%) \\ 14 \text { Gy } & 8(13 \%) \\ <14 \text { Gy } & 6(11 \%)\end{array}$

En nuestra serie registramos tres casos con complicaciones agudas $(5.1 \%)$ que consistieron en la aparición de crisis convulsivas. Las complicaciones demoradas o a largo plazo ocurrieron en siete casos (11.8\%) y consistieron en aumento de la clínica previa convulsiva ( 2 casos), aparición de de hiperintensidad en el T2 con efecto de masa (4 casos) y sangrado intraparenquimatoso (3 casos). Todos los pacientes con hiperintensidad y efecto de masa secundario respondieron al tratamiento esteroideo, sin precisar ninguna terapia más agresiva. De los pacientes con sangrado, dos precisaron de una evacuación urgente del hematoma mediante craneotomía. Dos pacientes fallecieron durante el seguimiento. Sólo una de las muertes fue consecuencia de sangrado de la MAV, mientras que la otra se debió a otra enfermedad no relacionada (carcinoma de mama).

El seguimiento total osciló entre 6 meses y 6 años (mediana de seguimiento 2.8 años). La tasa de oclusión a los 5 años fue del $77 \%$. Es destacable la importante proporción de casos en la que la oclusión se observó después de transcurridos 3 años de seguimiento (Fig 1)

En el análisis univariable de los diversos factores posiblemente relacionados con la tasa de oclusión no encontramos relación estadísticamente significativa con el sexo, presencia de sangrado o cirugía previos al tratamiento, la puntuación en la escala de Karnofsky, la localización ni la presencia de drenaje venoso profundo. La embolización previa y el grado de S-M mostraron una tendencia a la significación (P 0.1), en tanto que el tamaño y la hiperintensidad perilesional en T2 en RM se asociaron de manera estadísticamente significativa con la probabilidad de oclusión ( $\mathrm{P}=0.03$ y $\mathrm{P}=0.02$ respectivamente). En el estudio multivariable mediante regresión de Cox sólo el diámetro del nidus y la presencia de hiperintensidad en T2 en las RMs de control fueron factores independientes que se asociaron de manera estadísticamente significativa con la oclusión completa (Fig 2).

\section{Discusión}

Analizando los hallazgos en nuestra serie encontramos que la hiperintensidad perilesional en las secuencias T2 de las RMs de seguimiento y un diámetro del nidus menor de $3 \mathrm{~cm}$ son los dos factores con mayor implicación pronóstica en cuanto a la probabilidad de obliteración de las MAVs tratadas mediante radiocirugía. La aparición en las RMs de control de hiperintensidad perilesional en secuencias T2 ya ha sido descrita por diversos autores, hallazgo que ha sido atribuido a la aparición de edema vasogénico en la fase aguda como respuesta de los vasos a la radiación. Es por tanto posible postular que, a mayor exposición de los 


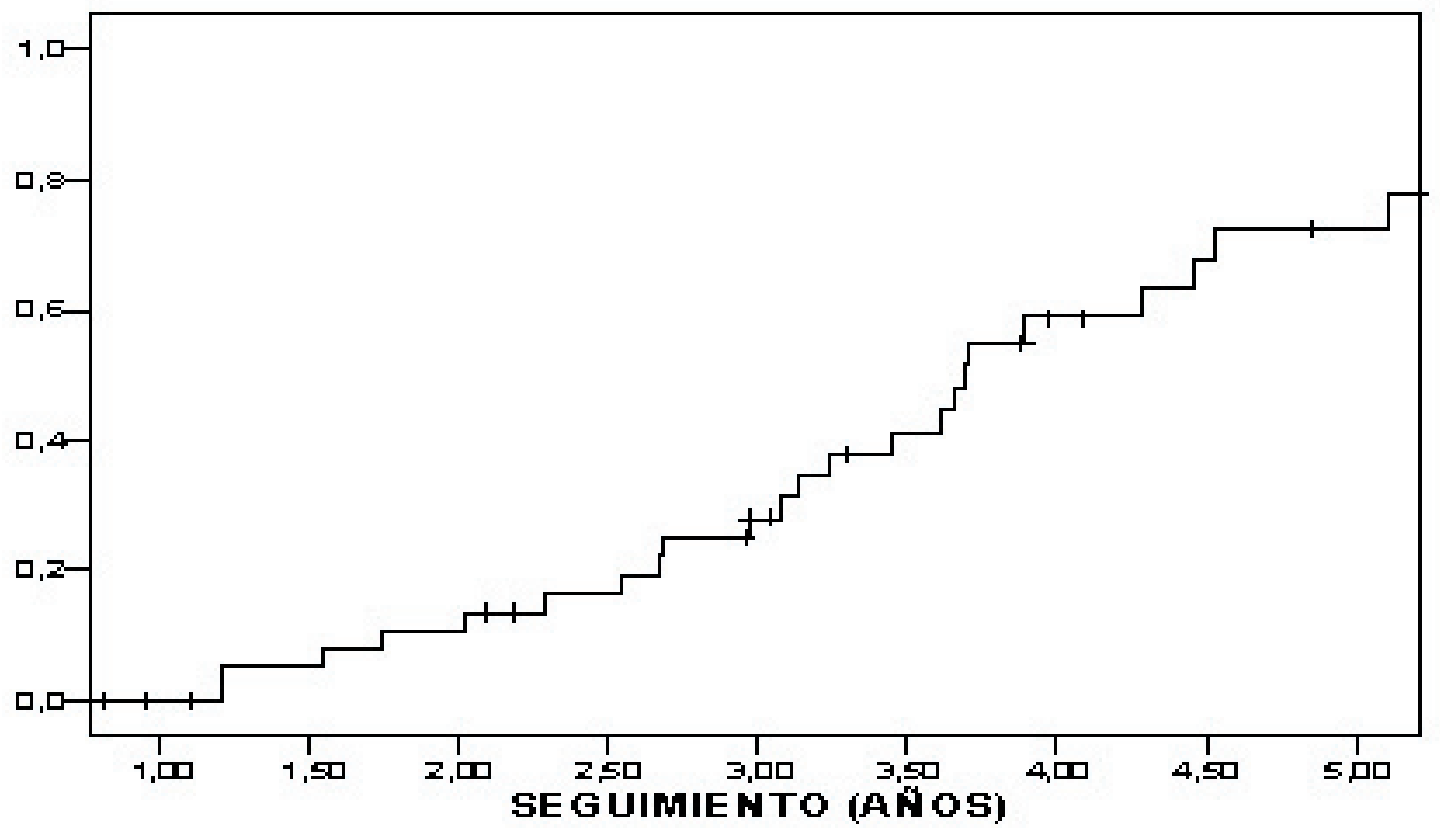

Figura 1. Probabilidad de oclusión de las MAVs en función del tiempo transcurrido tras el tratamiento radioquirúrgico.

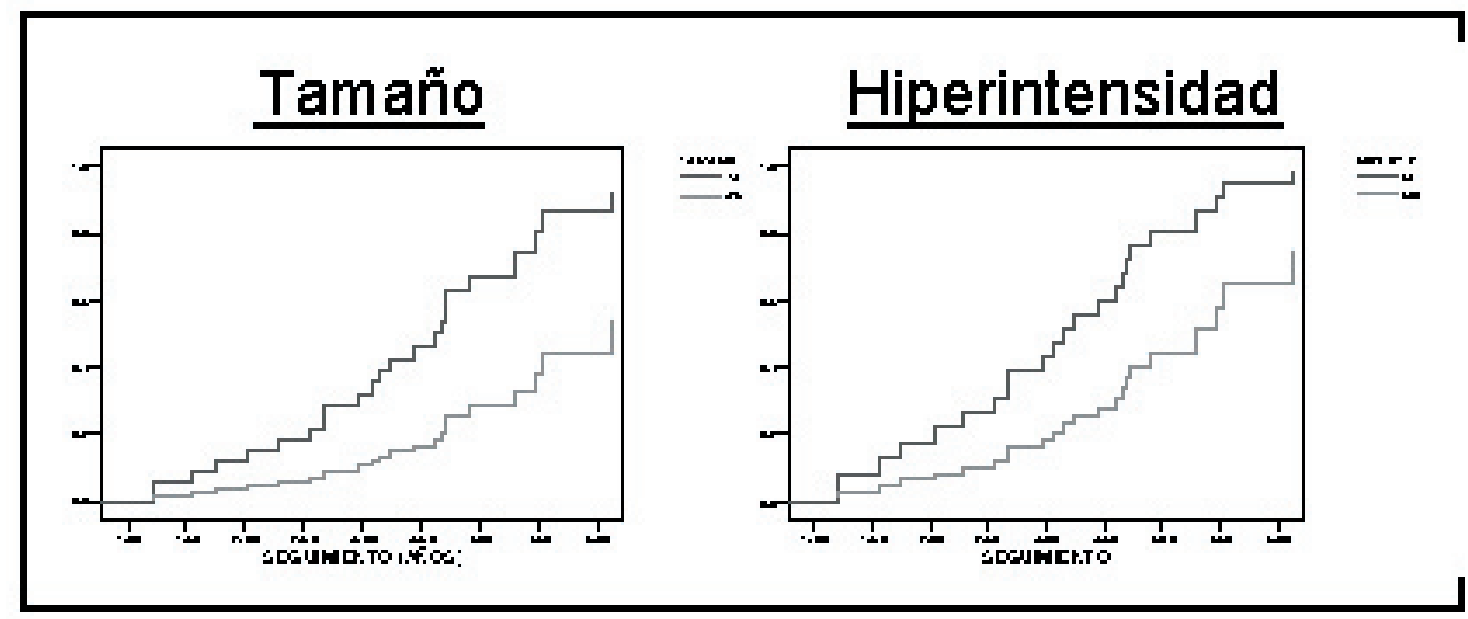

\begin{tabular}{|l|c|c|c|c|}
\hline & RR & P & \multicolumn{2}{|c|}{ IC (95\%) } \\
\hline Tamaño $<\mathbf{3 c m}$ & 3.3 & 0.05 & 0.973 & 11.499 \\
\hline Hiperintensidad T2 & 2.8 & 0.03 & 1.104 & 6.929 \\
\hline
\end{tabular}

Figura 2. Incremento de la probabilidad de oclusión completa de la MAV relacionada con un diámetro del nidus $<3 \mathrm{~cm}$ y con la aparición de hiperintensidad perilesional en secuencias T2 en las RMs de control.

vasos a la radiación en relación con un mayor tamaño del nidus, el riesgo teórico de generar edema resulta también mayor. En consecuencia, la aparición de estos cambios de señal podría traducir la mayor dosis de radiación recibida por los vasos nidales y perinidales con la consiguiente reacción tisular. Mobin y cols ${ }^{14}$ calcularon en su serie retrospectiva la sensibilidad y especificidad de la aparición de hiperintensidad en señales T2 como factores predictivos 
de obliteración, encontrando cifras del $72 \%$ y del $70 \%$ respectivamente. En cuanto al tamaño del nidus, aunque hay series en la literatura en las que se reportan porcentajes de obliteración de casi el $80 \%$ en MAVs con volúmenes mayores de $10 \mathrm{cc}^{20,21,25}$, en nuestra serie, las MAVs con diámetros menores de $3 \mathrm{cms}$ se obliteran en más del $80 \%$ de los casos frente a tan sólo un $50 \%$ en las de mayor tamaño $(\mathrm{P}<0.05)$.

Otro de lo datos a tener en cuenta, es la importante proporción de MAVs en las que hemos observado obliteración pasados los tres años tras el tratamiento radioquirúrgico. Es un hecho a recalcar, dado que la "frontera" de los tres años es la utilizada en muchas series para considerar el tratamiento como exitoso o fallido9. En nuestra serie, sin embargo, como se puede apreciar en la Fig.1, cerca de un $40 \%$ de las MAVs se obliteraron en el periodo comprendido entre los tres y los cinco años tras el tratamiento. Este dato tiene importantes implicaciones en el manejo de pacientes con MAV no ocluidas después de tres años tras el tratamiento puesto que, si sabemos que un importante porcentaje de MAVs pueden todavía obliterarse, parece más prudente mantener una actitud expectante con estos pacientes que recurrir a una nueva terapia agresiva. Esta actitud conservadora estaría especialmente indicada en pacientes con restos de MAVs de pequeño tamaño, en los cuales existen mayores posibilidades de obliteración.

Es preciso recordar, sin embargo, que cuando se elige el tratamiento radioquirúrgico de una MAV, durante un largo periodo de tiempo persiste el riesgo de hemorragia ${ }^{3}$. En nuestra serie, tres pacientes $(5 \%)$ presentaron hemorragias intraparenquimatosas durante el seguimiento, dos de los cuales precisaron craniotomía evacuadora y con resultado de muerte en uno de ellos. Existe cierta controversia en la literatura sobre si la radiocirugía disminuye o no el riesgo de sangrado en el periodo de tiempo transcurrido entre el tratamiento y la obliteración completa del nidus ${ }^{12,18,24}$. Por contra, parecen bien establecidas las características que incrementan el riesgo de hemorragia en la historia natural de las MAVs. Así, en la serie de Pollock y cols. ${ }^{17}$, el sangrado previo, el drenaje único y la morfología difusa en la arteriografía, eran marcadores estadísticamente significativos de un mayor riesgo de hemorragia. Por ello, la actitud seguida en nuestro centro es la de no realizar tratamientos radioquirúrgicos sobre MAVs con sangrado previo, salvo en casos de muy alto riesgo quirúrgico. En nuestra serie, sólo un 39\% de los pacientes tratados tenían como síntoma de presentación el sangrado intraparenquimatoso.

La embolización es un procedimiento ampliamente empleado para la reducción del tamaño de MAVs a fin de hacerlas subsidiarias de un posterior tratamiento quirúrgico o radioquirúgico ${ }^{4,5,6,7,10,22}$. En nuestra serie, en el análisis univariable, la embolización previa parecía ser un factor pronóstico para el fracaso en el tratamiento. Sin embargo, dado que las MAVs que precisan dicho procedimiento suelen ser obviamente las de mayor tamaño, al realizar el análisis multivariable ajustando por tamaño, la embolización previa perdía la significación estadística. Nuestros datos coinciden con los resultados de otras series de la literatura y parecen indicar que la posibilidad de oclusión de las MAVs radiadas tras embolización es similar a la de las MAVs no embolizadas que presenten un similar tamaño en el momento del procedimiento radioquirúgico ${ }^{2}$. Por ello, la embolización de MAVs cuyo tamaño supera los márgenes terapéuticos de la radiocirugía, con objeto de hacerlas subsidiarias de dicho tratamiento, parece una técnica adecuada según los datos obtenidos por nosotros.

La utilidad del grado de Spetzler-Martin como factor pronóstico en las series de MAVs tratadas con radiocirugía es muy discutida. Dicha escala fue creada para evaluar el riesgo quirúrgico de las MAVs y, por lo tanto, no parece lógico su empleo en series en las que el tratamiento presenta una problemática distinta. En nuestra serie el grado de Spetzler-Martin, cuando es ajustado por el tamaño de la MAV, no presenta influencia estadísticamente significativa sobre la probabilidad de oclusión, mostrando una vez más su poca utilidad como instrumento de predicción cuando se utiliza en este tipo de tratamiento. Polloc y cols. ${ }^{16}$ trataron de solventar este problema proponiendo un sistema de graduación de las MAVs adaptado al pronóstico radioquirúrgico tras tratamiento con Gamma Knife, pero este sistema apenas ha sido posteriormente empleado en otros estudios. En un reciente trabajo se ha confirmado la utilidad del sistema propuesto por Polloc y cols cuando el tratamiento radioquirúrgico se realiza con acelerador de electrones $\mathrm{y}$, además, se ha mostrado que la escala de Spetzler-Martin modificada por Oliveira y cols sí podría tener un valor predictivo para la tasa de oclusiones en radiocirugía ${ }^{1}$. El interés de esta observación estriba en que, de existir una escala que demostrase su valor predictivo tanto en el tratamiento microquirúrgico como en el radioquirúrgico, se podría comparar la utilidad de ambos procedimientos.

Aunque el objetivo de nuestro estudio no es comparar el tratamiento radioquirúrgico con el microquirúrgico, si se repasan los datos de series en las que las MAVs son tratadas microquirúrgicamente, se puede observar que los resultados no son sustancialmente diferentes ${ }^{15,23}$. Por ello, dado que la resección microquirúrgica ofrece como gran ventaja la desaparición inmediata del riesgo de sangrado, la política en nuestro centro es emplear el tratamiento radioquirúrgico preferentemenmte en las MAVs localizadas en áreas elocuentes, más aún si el nidus es $<3 \mathrm{cms}$ de diámetro y que no ha ocurrido sangrado.

\section{Bibliografía}

1. Andrade-Souza, Y.M., Zadeh, G., Ramani, M. et al.: 
Testing the radiosurgery-based arteriovenous malformation score and the modified Spetzler-Martin grading system to predict radiosurgical outcome. J. Neurosurg. 2005; 103: 642-648.

2. Bollet, M.A., Anxionnat, R., Buchheit, I. et al.: Efficacy and morbidity of arc-therapy radiosurgery for cerebral arteriovenous malformations: a comparison with the natural history. Int. J. Radiat. Oncol. Biol. Phys. 2004; 58: 1353-1363.

3. Brown, R.D., Jr., Flemming, K.D., Meyer, F.B. et al.: Natural history, evaluation, and management of intracranial vascular malformations .Mayo Clin.Proc. 2005; 80: 269-281.

4. Deruty, R., Pelissou-Guyotat, I., Amat, D. et al: Complications after multidisciplinary treatment of cerebral arteriovenous malformations. Acta Neurochir. (Wien.) 1996; 138: 119-131.

5. Deruty, R., Pelissou-Guyotat, I., Mottolese, C. et al.: The combined management of cerebral arteriovenous malformations. Experience with 100 cases and review of the literature. Acta Neurochir. (Wien.) 1993; 123: 101-112.

6. Duffner, F., Freudenstein, D., Becker, G. et al.: Combined treatment effects after embolization and radiosurgery in high-grade arteriovenous malformations. Case report and review of the literature. Stereotact. Funct. Neurosurg. 2000; 75: 27-34.

7. Firlik, A.D., Levy, E.I., Kondziolka, D. et al.: Staged volume radiosurgery followed by microsurgical resection: a novel treatment for giant cerebral arteriovenous malformations: technical case report. Neurosurgery 1998; 43: 1223-1228.

8. Flickinger, J.C., Kondziolka, D., Maitz, A.H. et al.: An analysis of the dose-response for arteriovenous malformation radiosurgery and other factors affecting obliteration. Radiother. Oncol. 2002; 63: 347-354.

9. Gallina, P., Merienne, L., Meder, J.F. et al.: Failure in radiosurgery treatment of cerebral arteriovenous malformations. Neurosurgery 1998; 42: 996-1002.

10. Henkes, H., Nahser, H.C., Berg-Dammer, E. et al.: Endovascular therapy of brain AVMs prior to radiosurgery. Neurol. Res. 1998; 20: 479-492.

11. Maesawa, S., Flickinger, J.C., Kondziolka, D. et al: Repeated radiosurgery for incompletely obliterated arteriovenous malformations. J. Neurosurg. 2000; 92: 961-970.

12. Maruyama, K., Kawahara, N., Shin, M. et al.: The risk of hemorrhage after radiosurgery for cerebral arteriovenous malformations. N. Engl. J. Med. 2005; 352: 146-153.

13. Maruyama, K., Kondziolka, D., Niranjan, A. et al.: Stereotactic radiosurgery for brainstem arteriovenous malformations: factors affecting outcome. J. Neurosurg. 2004; 100: 407-413.

14. Mobin, F., De Salles, A.A., Abdelaziz, O. et al.: Stereotactic radiosurgery of cerebral arteriovenous malformations: appearance of perinidal $\mathrm{T}(2)$ hyperintensity signal as a predictor of favorable treatment response. Stereotact. Funct. Neurosurg. 1999; 73: 50-59.

15. Pikus, H.J., Beach, M.L., Harbaugh, R.E.: Microsurgical treatment of arteriovenous malformations: analysis and comparison with stereotactic radiosurgery. J. Neurosurg. 1998; 88: 641-646.

16. Pollock, B.E., Flickinger, J.C.: A proposed radiosurgery-based grading system for arteriovenous malformations. J. Neurosurg. 2002; 96: 79-85.

17. Pollock, B.E., Flickinger, J.C., Lunsford, L.D. et al.: Factors that predict the bleeding risk of cerebral arteriovenous malformations. Stroke 1996; 27: 1-6.

18. Pollock, B.E., Flickinger, J.C., Lunsford, L.D. et al.: Hemorrhage risk after stereotactic radiosurgery of cerebral arteriovenous malformations. Neurosurgery 1996; 38: 652659.

19. Pollock, B.E., Flickinger, J.C., Lunsford, L.D. et al.: Factors associated with successful arteriovenous malformation radiosurgery. Neurosurgery 1998; 42: 1239-1244.

20. Pollock, B.E., Kline, R.W., Stafford, S.L. et al.: The rationale and technique of staged-volume arteriovenous malformation radiosurgery. Int. J. Radiat. Oncol. Biol. Phys. 2000; 48: 817-824.

21. Pollock, B.E., Kondziolka, D., Lunsford, L.D. et al.: Repeat stereotactic radiosurgery of arteriovenous malformations: factors associated with incomplete obliteration. Neurosurgery 1996; 38: 318-324.

22. Pollock, B.E., Lunsford, L.D., Kondziolka, D. et al.: Embolization and radiosurgery for AVMs. J. Neurosurg. 1997; 86: 319-320.

23. Pollock, B.E., Lunsford, L.D., Kondziolka, D. et al.: Patient outcomes after stereotactic radiosurgery for "operable" arteriovenous malformations. Neurosurgery 1994; 35: 1-7.

24. Shin, M., Kawahara, N., Maruyama, K. et al.: Risk of hemorrhage from an arteriovenous malformation confirmed to have been obliterated on angiography after stereotactic radiosurgery. J. Neurosurg. 2005; 102: 842-846.

25. Yamamoto, Y., Coffey, R.J., Nichols, D.A. et al.: Interim report on the radiosurgical treatment of cerebral arteriovenous malformations. The influence of size, dose, time, and technical factors on obliteration rate. J. Neurosurg. 1995; 83: 832-837.

Arrese, I.; Alday, R.; González, P.A.; Campollo, J.; Ramos, A.; Dominguez, P.; Fernandez-Letón, J.P.; Pérez-Nuñez, A.; Lobato, R.D.: Hiperintensidad en secuencias T2 y tamaño como factores pronósticos de obliteración en malformaciones arteriovenosas tratadas mediante radiocirugía. Neurocirugçia 2009; 20: 97-102

Correspondencia postal: Ignacio Arrese Regañón. Servicio de Neurocirugía. Hospital 12 de Octubre. Avda. de Córdoba s/n. 28041 Madrid. Spain. 